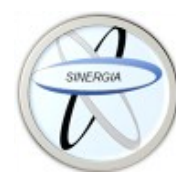

Revista Médica Sinergia

https://doi.org/10.31434/rms.v6i11.718

Vol. 6, Núm. 11, noviembre. 2021,

$\underline{\mathrm{e} 718}$

revistamedicasinergia@gmail.com

\title{
Manejo de la osteoporosis en la edad posmenopáusica
}

Management of osteoporosis in the postmenopausal age

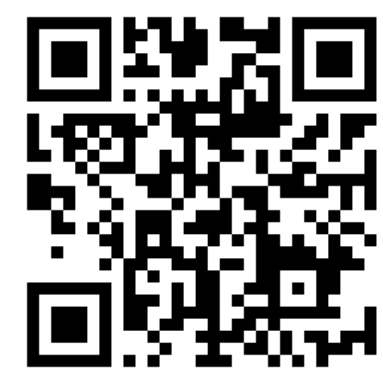

Recibido $26 / 07 / 2021$
${ }^{1}$ Dra. Saskia Sharielle Franken Morales Investigadora independiente, Cartago, Costa Rica

(iD) https://orcid.org/0000-0002-5496-9218

${ }^{2}$ Dra. Ana Maria Garcia Orrego Emergencias Médicas del Continente, San José, Costa Rica

(iD https://orcid.org/0000-0002-2255-8107

${ }^{3}$ Dr. Luis Valenzuela Barrantes Hospital Dr. Rafael Ángel Calderón Guardia, San José, Costa Rica

(D) https://orcid.org/0000-0002-2247-4210

Corregido $10 / 08 / 2021$ 20/08/2021

\section{RESUMEN}

La osteoporosis es una enfermedad caracterizada por un aumento en el recambio óseo y disminución de la masa ósea asociada a fragilidad esquelética, lo que aumenta el riesgo de caídas y fracturas. Inherente a su presentación, la osteoporosis es asintomática hasta que ocurren las fracturas. Actualmente existe una variedad de medicamentos que pueden ser utilizados en la osteoporosis por lo que es importante conocer cada uno de ellos e individualizar cada paciente para saber cuándo y a quiénes brindar terapia farmacológica. Los más utilizados son los inhibidores de la resorción ósea, los agentes anabólicos, la calcitonina, los moduladores selectivos del receptor de estrógeno y el Denosumab. El desafío en cuanto a esta patología es lograr captar mujeres con alto riesgo para así establecer una terapia de forma temprana con el objetivo de prevenir fracturas.

PALABRAS CLAVE: osteoporosis; densidad mineral ósea; posmenopausia; bifosfonatos.

\section{ABSTRACT}

Osteoporosis is a disease characterized by increased bone turnover and decreased bone mass associated with skeletal fragility, which increases the risk of falls and fractures. Inherent in this presentation, osteoporosis is asymptomatic until fractures occur. Currently there is a variety of 
drugs that can be used in osteoporosis, so it is important to know each one of them and individualize each patient to know when and to whom to provide drug therapy. The most widely used are bone resorption inhibitors, anabolic agents, calcitonin, selective estrogen receptor modulators, and Denosumab. The challenge regarding this pathology is to attract women at high risk in order to establish an early therapy with the aim of preventing fractures.

KEYWORDS: osteoporosis; bone density; postmenopause; bisphosphonates.

${ }^{1}$ Médica general, graduada de la Universidad de Ciencias Médicas (UCIMED). Cód. MED16453. Correo: sharielle franken@hotmail.com

${ }^{2}$ Médica general, graduada de la Universidad de Ciencias Médicas (UCIMED). Cód. MED16113. Correo: anamariag17@hotmail.com

${ }^{3}$ Médico general, graduado de la Universidad de Ciencias Médicas (UCIMED). Cód. MED12951. Correo: Ivb2889@gmail.com

\section{INTRODUCCIÓN}

La osteoporosis es una afección crónica y progresiva caracterizada por una disminución de la masa ósea con deterioro de la microarquitectura (1). Sus consecuencias incluyen una mayor fragilidad ósea y un alto riesgo de fracturas. La incidencia aumenta con el envejecimiento y afecta aproximadamente al $30 \%$ de las mujeres posmenopáusicas (2). Según la Organización Mundial de la Salud (OMS), la osteoporosis es la segunda patología más común después de las enfermedades cardiovasculares. Aunque la enfermedad afecta tanto a mujeres como a hombres, la forma más común, que constituye aproximadamente el $90 \%$ de los casos, es la osteoporosis posmenopáusica (3). Aunque la osteoporosis se ha diagnosticado tradicionalmente sobre la base de una densidad ósea baja en ausencia de fractura, las Guías de práctica clínica para el diagnóstico y tratamiento de las osteoporosis posmenopáusicas de 2016 y 2020, indican que la osteoporosis también puede ser diagnosticada en pacientes con osteopenia y un mayor riesgo de fractura (3). La osteoporosis es un factor importante que contribuye a la disminución de la esperanza de vida y al deterioro significativo de la calidad de vida de los adultos mayores (1) por lo que es crucial realizar un diagnóstico de forma temprana.

Esta revisión tiene como objetivo identificar las mujeres con alto riesgo de fractura y dirigir la terapia farmacológica de forma individualizada.

\section{MÉTODO}

Para la presente revisión bibliográfica, se realizó una búsqueda en las siguientes bases de datos: UpToDate, Cochrane, PubMed, Scielo, Elsevier, y ScienceDirect. Se revisaron 22 artículos entre los años 2016 al 2021.

Finalmente se utilizaron 16 bibliografías publicadas entre los años 2017 y 2021 procedentes de países como Bélgica, Brasil, China, Estados Unidos, Francia, Grecia, Suiza y Unión Europea. Se utilizaron artículos de estudio de casos y controles, metaanálisis, publicación de casos, estudios observacionales y revisión bibliográfica en inglés. Se incluyeron investigaciones que contuviera información pertinente en cuanto a epidemiología, fisiopatología, herramientas diagnósticas, y tratamiento farmacológico.

Finalmente se realizó un análisis de la información recolectada, con énfasis en los aspectos más novedosos de esta enfermedad. 


\section{EPIDEMIOLOGÍA}

La osteoporosis representa un importante problema de salud pública en todo el mundo. Según la OMS causa más de 8,9 millones de fracturas en todo el mundo. La probabilidad de por vida de fracturas osteoporóticas como la cadera, muñeca y vertebral- es del 30 al $40 \%$ y con una población que envejece, se espera que la prevalencia de la osteoporosis solo siga aumentando. De las personas que sufren una fractura, solamente el $40 \%$ logra recuperar completamente la movilidad. Además, muchas personas no reciben tratamiento una vez que han tenido fracturas osteoporóticas. Solo el $23 \%$ de las mujeres de 67 años o más reciben una prueba o prescripción de densidad mineral ósea dentro de los 6 meses posteriores a la fractura (4).

Muchos estudios muestran que el riesgo de fracturas en pacientes osteoporóticos puede reducirse en un $70 \%$ con solamente utilizar terapia de protección ósea (2).

\section{FISIOPATOLOGÍA}

El proceso de resorción ósea está controlado por una compleja interacción entre las células osteoblásticas y osteoclásticas. La osteoporosis es causada por un desequilibrio entre estos dos procesos biológicos, lo que conduce a una pérdida ósea generalizada. Las células encargadas de este proceso son los osteoclastos, que expresan el receptor activador del factor nuclear kB (RANK). El ligando del receptor activador del factor nuclear $\mathrm{kb}$ (RANKL) se libera por los osteoblastos y se une al RANK a nivel del osteoclasto inactivo. Esta unión da como resultado la osteoclastogénesis. Por otro lado, el sistema de señalización de osteoprotegerina (OPG) previene la progresión de esta vía al competir con RANKL para unirse al RANK, previniendo así la osteoclastogénesis. En pocas palabras, la reabsorción ósea impulsada por los osteoclastos está mediada por el desequilibrio RANKL-OPG, donde el aumento de RANKL conduce a un aumento de la osteoclastogénesis y la consiguiente pérdida ósea. La liberación de RANKL está regulada por varias citocinas y hormonas (4).

\section{FACTORES DE RIESGO}

\section{$\underline{\text { Hormonales }}$}

Los estrógenos suprimen la actividad osteoclástica (4). En las mujeres posmenopáusicas la deficiencia de estrógenos se asocia con una mayor producción y liberación de citocinas con el subsecuente aumento del número y actividad osteoclástica. Esto a su vez estimula una mayor resorción ósea (5).

Se ha visto que la hormona paratiroidea (PTH) aumenta la actividad osteoclástica por lo que el hiperparatiroidismo primario se asocia con cierta pérdida ósea. También con el envejecimiento se da una disminución en la ingesta de calcio, en la absorción intestinal de calcio, exposición a la luz solar y de la capacidad de la piel para producir vitamina D.

El estado de hiperparatiroidismo secundario crónico derivado de las deficiencias de calcio y vitamina $D$ aumenta el recambio óseo y favorece un balance óseo negativo, y con esto osteoporosis (5).

\section{$\underline{\text { Nutricionales }}$}

La deficiencia nutricional juega un papel importante en la osteoporosis en los adultos mayores. La desnutrición y, en particular, la desnutrición proteico-energética es un factor 
de riesgo de osteoporosis, sarcopenia y fragilidad (5).

Se requieren suministros adecuados de proteína dietética para un crecimiento óseo óptimo y el mantenimiento de huesos sanos. En los adultos mayores con osteoporosis, una ingesta mayor a $0,8 \mathrm{~g}$ por $\mathrm{kg}$ de peso corporal al día se asocia con una mayor densidad mineral ósea, menor tasa de pérdida ósea y menor riesgo de fractura de cadera. Esto siempre y cuando la ingesta dietética de calcio sea la adecuada (6).

\section{Toxicológicos}

El tabaquismo genera cambios a nivel de la microarquitectura trabecular, causando reducción de resistencia ósea al estrés mecánico.

En un estudio con 100 mujeres se demostró que la prevalencia de osteoporosis fue mucho mayor en el grupo de fumadores $(31,3 \%)$ frente a exfumadores $(28,6 \%)$ o no fumadores $(7,5 \%)$.

El alcohol contiene ciertas sustancias que asemejan el efecto estimulante del estrógeno a nivel de los huesos por lo que se ha visto que el consumo de una bebida alcohólica al día, en especial vino, tiene un efecto ligeramente positivo sobre la densidad mineral ósea.

Por otro lado, el consumo excesivo de alcohol tiene un impacto negativo sobre la homeostasis ósea. También se logró demostrar que la ingesta de cafeína conduce a una disminución en la absorción intersticial de calcio, y las dosis altas de cafeína -más de 4 tazas al día- pueden acelerar la pérdida ósea a nivel de la columna lumbar en mujeres posmenopáusicas mayores (7).

\section{Otros factores asociados}

Edad, raza blanca, menopausia precoz, amenorrea primaria $\mathrm{O}$ secundaria, antecedente de fractura, bajo índice de masa corporal, trastornos neuromusculares, tratamiento con glucocorticoides.

\section{DIAGNÓSTICO}

\section{Absorciometría de rayos $X$ de energía dual}

La absorciometría de rayos $X$ de energía dual (DXA) se considera el estándar de oro para medir la densidad mineral ósea (DMO). Los resultados de la DMO se informan como puntuaciones $\mathrm{T}$, este es el número de desviaciones estándar (DE) del valor de DMO medido por encima o por debajo de la DMO media en mujeres jóvenes. La osteoporosis se define como una puntuación T menor o igual $-2,5$ en el cuello femoral. Una puntuación $T$ entre $-1,0$ a $\quad-2,5$ osteopenia y mayor $-1,0$ se define como normal (8). La DXA a nivel del cuello femoral es el sitio preferido para generar una puntuación $\mathrm{T}$, debido a su alto valor predictivo para riesgo de fractura. Los cambios degenerativos en la columna, que son comunes en pacientes de edad avanzada, pueden elevar falsamente la DMO, por esta razón la columna no es el sitio preferido para el diagnóstico de osteoporosis en mujeres posmenopáusicas. No obstante, el cálculo de la DMO en la columna es el sitio preferido para evaluar la respuesta al tratamiento $(4,11)$. La limitación de la DMO es que la mayoría de las fracturas ocurren en individuos con una puntuación $\mathrm{T}$ que no cumple con la definición de osteoporosis y, por lo tanto, tiene baja sensibilidad cuando se usa solo para la detección de osteoporosis $(9,11)$.

Se debe considerar la evaluación de fracturas vertebrales si hay una puntuación T de DMO menor o igual a -2,5 (12). 


\section{Herramienta de Evaluación de Riesgo de} Fractura

La identificación de las mujeres de alto

riesgo tiene como objetivo evitar la primera fractura por fragilidad. Las mujeres posmenopáusicas deben ser valoradas anualmente con la Herramienta de Evaluación de Riesgo de Fractura (FRAX). El FRAX es una calculadora que evalúa el riesgo de fracturas osteoporóticas a 10 años basándose en factores de riesgo individuales, con o sin valores de DMO (10). La interpretación de las puntuaciones FRAX puede verse influida por la exposición a glucocorticoides, la información sobre la DMO de la columna lumbar, la puntuación del hueso trabecular, la longitud del eje de la cadera, el historial de caídas, el estado migratorio y la diabetes mellitus tipo 2. Se debe considerar la evaluación de fracturas vertebrales si hay antecedentes de pérdida de altura $\geq 4 \mathrm{~cm}$, cifosis, tratamiento con glucocorticoides orales reciente o actual a largo plazo (12).

\section{TRATAMIENTO}

\begin{tabular}{llll} 
El objetivo de & utilizar terapias \\
farmacológicas & \multicolumn{2}{c}{ en } & mujeres \\
posmenopáusicas es disminuir la
\end{tabular}
probabilidad de fracturas osteoporóticas. La terapia farmacológica está indicada en pacientes con puntajes $\mathrm{T}$ en el rango osteoporótico y aquellos con antecedentes de fractura por fragilidad. Sin embargo, es importante señalar que la mayoría de las fracturas se producen en pacientes con osteopenia (12,13). Antes de iniciar el tratamiento, es apropiado estratificar el riesgo de cada paciente, porque esto puede influir en la selección del tratamiento inicial, así como en la duración de la terapia (13). Se trata a:
- Se recomienda la terapia farmacológica para pacientes con osteopenia o baja masa ósea y antecedentes de fractura por fragilidad de la cadera o la columna (Grado A1) (14).

- Pacientes con una puntuación T de -2,5 o inferior en la columna, el cuello femoral, la cadera total o $1 / 3$ del radio (Grado A1) (14).

- Pacientes con una puntuación T entre 1,0 y $-2,5$ si el FRAX a 10 años para la fractura osteoporótica es $\geq 20 \%$ o la probabilidad de fractura de cadera a 10 años es $\geq 3 \%$ o por encima del umbral específico según el país (Grado A1) (14).

\section{Farmacoterapia}

- Bifosfonatos: son la clase de medicación más utilizada para el tratamiento de la osteoporosis. Una revisión sistemática de ensayos publicados entre 2005 y 2019 confirmó la eficacia en la prevención de fracturas vertebrales de alendronato, risedronato, ácido zoledrónico e ibandronato, en comparación con placebo. El alendronato, risedronato y ácido zoledrónico también reducen el riesgo de cadera y otras enfermedades no vertebrales (13). Se recomienda que el riesgo de fracturas se reevalúe después de 3 a 5 años de tratamiento con bifosfonatos. Las mujeres que permanecen en alto riesgo de fracturas deben continuar el tratamiento, mientras que aquellas que tienen un riesgo de fracturas bajo a moderado deben de realizar la suspensión temporal del tratamiento. Se recomienda reevaluar el riesgo de fractura en intervalos de 2 a 4 años y se debe de considerar reiniciar la terapia para la osteoporosis antes de un máximo sugerido de 5 años si hay una 
disminución significativa en la densidad mineral ósea, una fractura intermedia $u$ otros factores que alterar el estado de riesgo clínico (16).

- Raloxifeno: es un modulador selectivo del receptor de estrógenos, con efectos agonistas sobre los huesos. Está aprobado por la FDA para la prevención y el tratamiento de la osteoporosis posmenopáusica, así como para la reducción del riesgo de cáncer de mama en mujeres con osteoporosis posmenopáusica o con alto riesgo de cáncer de mama. El principal ensayo de eficacia del raloxifeno fue el ensayo de Múltiples Resultados de la Evaluación de Raloxifeno (MORE). Se ha demostrado que el Raloxifeno reduce el riesgo de fractura vertebral. No se observaron diferencias significativas en la reducción de fracturas no vertebrales y de cadera (13). En mujeres posmenopáusicas con osteoporosis con alto riesgo de fractura, bajo riesgo de trombosis venosa profunda o tienen un alto riesgo de cáncer de mama se recomienda el raloxifeno (16).

- Calcitonina: es más útil como agente alternativo después de una fractura vertebral aguda debido a sus efectos analgésicos sistémicos. Tiene un efecto modesto sobre la DMO y la reducción de fracturas, y se recomienda su uso con un antirresortivo más fuerte cuando sea posible (13). En mujeres posmenopáusicas con alto riesgo de fractura con osteoporosis, se sugiere que la calcitonina en aerosol nasal debe prescribirse únicamente a mujeres que no pueden tolerar los bifosfonatos, el Raloxifeno, los estrógenos, la teriparatida y el denosumab (16).
- Estrógenos: alguna vez se consideró el tratamiento de elección para la osteoporosis posmenopáusica, sin embargo, nunca fue aprobado específicamente para este uso (13). Actualmente se recomienda en mujeres posmenopáusicas menores de 60 años o menos de 10 años de inicio de la menopausia con alto riesgo de fractura, con histerectomía, bajo riesgo de trombosis venosa profunda, con síntomas vasomotores adicionales; sin antecedentes de infarto de miocardio o accidente cerebrovascular previo, sin cáncer de mama y aquellas en los que los bifosfonatos o el Denosumab no son apropiados (16)

- Denosumab: está aprobado por la FDA para el tratamiento de la osteoporosis en mujeres posmenopáusicas, osteoporosis masculina, osteoporosis inducida por glucocorticoides y pérdida ósea inducida por el tratamiento del cáncer. El denosumab se administra como una inyección subcutánea de $60 \mathrm{mg}$ cada 6 meses (13). Los efectos del denosumab sobre la remodelación ósea, reflejados en los marcadores de recambio óseo, se revierten después de 6 meses si el fármaco no se toma según lo programado. Se han notificado casos de fracturas vertebrales múltiples al interrumpir el tratamiento, por lo tanto, no se recomienda debido a este posible aumento del riesgo de fractura $(15,16)$. Aunque se necesitan más datos para dilucidar aún más el impacto clínico de este fenómeno, se debe informar a los pacientes sobre la importancia de no omitir una dosis de denosumab y se debe evitar la interrupción del denosumab sin un plan de transición de tratamiento adecuado. Las pautas de la 
Asociación Americana de Endocrinólogos Clínicos / Colegio Americano de Endocrinología para la osteoporosis posmenopáusica recomendaban que las pacientes recibieran una transición con ácido zoledrónico intravenoso 0 , alternativamente, alendronato durante 1 año. Durante este período de transición, los marcadores de recambio óseo y la DXA pueden seguirse y los pacientes pueden monitorearse de cerca para detectar evidencia de aumento de rebote en la resorción ósea o fracturas vertebrales múltiples $(13,15,16)$.

- Teriparatida: aprobado para el tratamiento de la osteoporosis posmenopáusica y masculina por hasta 2 años para la reducción de fracturas vertebrales y no vertebrales. Una vez que se completa el ciclo de tratamiento, se recomienda el tratamiento con terapias antirresortivas para mantener la ganancia de densidad ósea $(13,16)$.

- Romosozumab: en mujeres posmenopáusicas con osteoporosis con riesgo muy alto de fractura, como aquellas con osteoporosis grave (es decir, puntuación $\mathrm{T}$ baja $<-2,5$ y fracturas) 0 fracturas vertebrales múltiples, se recomienda el tratamiento con romosozumab durante un máximo de 1 año para la reducción de fracturas de cadera y no vertebrales. Las mujeres con alto riesgo de enfermedad cardiovascular y accidente cerebrovascular no deben ser consideradas para el uso de romosozumab. Una vez completado un ciclo de romosozumab, se recomienda el tratamiento con terapias antirresortivas para mantener las ganancias de densidad mineral ósea y reducir el riesgo de fracturas (16).

\section{CONCLUSIONES}

El riesgo de sufrir una fractura por fragilidad aumenta con la edad debido a la disminución de la DMO y esto es un problema sanitario importante. El impacto de una fractura en la vida de los pacientes es inmenso y los costos para la sociedad también son altos, tanto en términos de atención como en la parte de rehabilitación. La prevención primaria de las fracturas por fragilidad es el enfoque más rentable y proactivo para mantener la salud ósea. En la actualidad existen suficientes herramientas que nos ayudan a estratificar el riesgo en cada paciente para así poder captar las que presentan un alto riesgo de cursar con una fractura. También es importante educar a las pacientes en cuanto a factores modificables que pueden tener un impacto negativo significativo sobre la densidad mineral ósea como el tabaquismo, la nutrición, la ingesta de alcohol y el ejercicio físico. La captación temprana de estas mujeres es imperativo para brindarles un tratamiento adecuado con el objetivo de reducir la fragilidad ósea y evitar las fracturas.

\section{Los autores declaran no tener conflicto de interés.}

\section{REFERENCIAS}

1. Zhu Y, Huang Z, Wang $Y, X u$ W. The efficacy and safety of denosumab in postmenopausal women with osteoporosis previously treated with bisphosphonates: A review. Journal of Orthopaedic Translation. 2020;22:7-9. Available from: https://doi.org/10.1016/j.jot.2019.08.004

2. Rossi L, Copes R, Dal Osto L, Flores C, Comim F, Premaor M. Factors related with osteoporosis treatment in postmenopausal women. Medicine. 
2018;97(28):1-2. Available from: http://dx.doi.org/10.1097/MD.0000000000011524

3. Anda S, Evija N, Andris A, Una S, Aivars L. Gray values of the cervical vertebrae detected by conebeam computed tomography for the identification of osteoporosis and osteopenia in postmenopausal women. Oral Surg Oral Med Oral Pathol Oral Radiol. 2021;:4-8. Available from: https://doi.org/10.1016/j.0000.2021.06.014

4. Shanks G, Sharma D, Mishra V. Prevention and treatment of osteoporosis in women. OBSTETRICS, GYNAECOLOGY AND REPRODUCTIVE MEDICINE. 2019;29(7):202204. Available from: https://doi.org/10.1016/j.ogrm.2019.04.001

5. Rizzoli R. Postmenopausal osteoporosis: Assessment and management. Best Practice \& Research Clinical Endocrinology \& Metabolism. 2018;32(5):740-741. Available from: https://doi.org/10.1016/j.beem.2018.09.005

6. Rizzoli, R., Biver, E., Bonjour, JP. et al. Benefits and safety of dietary protein for bone health-an expert consensus paper endorsed by the European Society for Clinical and Economical Aspects of Osteopororosis, Osteoarthritis, and Musculoskeletal Diseases and by the International Osteoporosis Foundation. Osteoporosis International. 2018;29:1933-1948 Available from: https://doi.org/10.1007/s00198-018-4534-5

7. Bijelic R, Milicevic S, Balaban J. Risk Factors for Osteoporosis in Postmenopausal Women. Med Arch. 2017;71(1):26-27. Available from: https://doi.org/10.5455/medarh.2017.71.25-28

8. Briot K, Roux C, Thomas T. 2018 update of French recommendations on the management of postmenopausal osteoporosis. Joint Bone Spine. 2018;85(5):521-525. Available from: https://doi.org/10.1016/j.jbspin.2018.02.009

9. Compston J, Leslie W, McClung M. Osteoporosis. Lancet. 2019;393:364-369.

10. French K, Emanuele D. Osteoporosis: Increasing Screening and Treatment for Postmenopausal Women. The Journal for Nurse Practitioners. 2019;15(5):349. Available from: https://doi.org/10.1016/j.nurpra.2019.02.014

11. Compston J, Cooper A, Cooper C. UK clinical guideline for the prevention and treatment of osteoporosis. Arch Osteoporos. 2017;12(1):4244. Available from: https://doi.org/10.1007/s11657-017-0324-5

12. Kanis J, Cooper C, Rizzoli R. European guidance for the diagnosis and management of osteoporosis in postmenopausal women. Osteoporosis International. 2019;30:4-6, 13-15.
Available from: https://doi.org/10.1007/s00198018-4704-5

13. Arceo-Mendoza R, Camacho P. Postmenopausal Osteoporosis: Latest Guidelines. Endocrinol Metab Clin N Am. 2021;50:167-176. Available from: https://doi.org/10.1016/j.ecl.2021.03.009

14. Camacho P, Petak S, Binkley N. AMERICAN ASSOCIATION OF CLINICAL ENDOCRINOLOGISTS/ AMERICAN COLLEGE OF ENDOCRINOLOGY CLINICAL PRACTICE GUIDELINES FOR THE DIAGNOSIS AND TREATMENT OF POSTMENOPAUSAL OSTEOPOROSIS- $2020 \quad$ UPDATE. ENDOCRINE PRACTICE. 2020;26(1):5-11. Available from: https://doi.org/10.4158/GL-20200524SUPPL

15. Anastasilakis A, Evangelatos G, Makras $P$, Iliopoulos A. Rebound-associated vertebral fractures may occur in sequential time points $T$ following denosumab discontinuation: need for prompt treatment re- initiation. Bone Reports. 2020;12:3. Available from: https://doi.org/10.1016/j.bonr.2020.100267

16. Shoback D, Rosen C, Black D, Cheung A. Pharmacological Management of Osteoporosis in Postmenopausal Women: An Endocrine Society Guideline Update. CLINICAL PRACTICE GUIDELINE UPDATE. 2020;105(3):2-3. Available from: https://doi.org/10.1210/clinem/dgaa048 